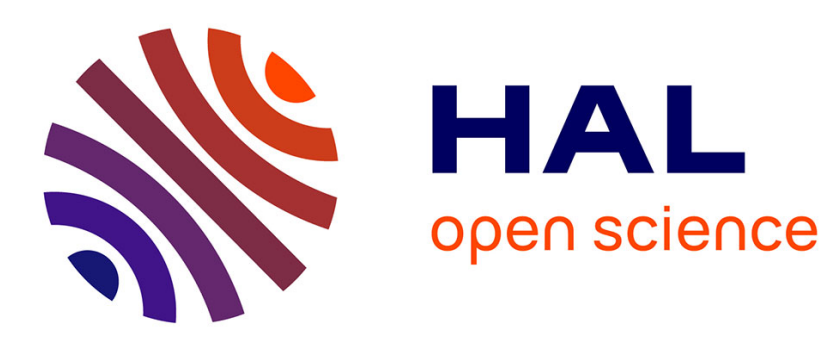

\title{
Static Properties of a Star Polymer in a High Molecular Weight Solvent
}

C. Gay, E. Raphaël

\section{To cite this version:}

C. Gay, E. Raphaël. Static Properties of a Star Polymer in a High Molecular Weight Solvent. Journal de Physique II, 1996, 6 (5), pp.587-591. 10.1051/jp2:1996199 . jpa-00248319

\section{HAL Id: jpa-00248319 https://hal.science/jpa-00248319}

Submitted on 1 Jan 1996

HAL is a multi-disciplinary open access archive for the deposit and dissemination of scientific research documents, whether they are published or not. The documents may come from teaching and research institutions in France or abroad, or from public or private research centers.
L'archive ouverte pluridisciplinaire HAL, est destinée au dépôt et à la diffusion de documents scientifiques de niveau recherche, publiés ou non, émanant des établissements d'enseignement et de recherche français ou étrangers, des laboratoires publics ou privés. 


\title{
Short Communication
}

\section{Static Properties of a Star Polymer in a High Molecular Weight Solvent}

\author{
C. Gay and E. Raphaël $\left(^{*}\right)$ \\ Laboratoire de Physique de la Matière Condensée $\left({ }^{* *}\right)$, Collège de France, \\ 11 Place Marcelin Berthelot, 75231 Paris Cedex 05, France
}

(Received 15 January 1996, received in final form 11 March 1996, accepted 20 March 1996)

PACS.36.20.Ey - Conformation (statistics and dynamics)

PACS.61.41.+e - Polymers, elastomers, and plastics

\begin{abstract}
Recently, Raphaël, Pincus and Fredrickson considered the static properties of an isolated star polymer ( $f$ arms, $N$ monomers per arm) dissolved in a melt of linear chains of degree of polymerization $P<N$, which are chemically identical to the star arms (Macromolecules 26 (1993) 1996). In the present article we show that the results obtained by these authors are valid only for stars with a high enough number of arms $f>P^{1 / 2}$ For $f<P^{1 / 2}$, a new Gaussian region appears within the star structure. We predict that the $N$-monomer volume fraction varies as $\phi(r) \cong 1$ (dense region) for $r<a f, \phi(r) \cong f(r / a)^{-1}$ for $a f<r<a P f^{-1}$ and $\phi(r) \cong P^{1 / 3} f^{2 / 3}(r / a)^{-4 / 3}$ for $r>a P f^{-1}$. Our study is restricted to scaling laws; the exact prefactors in our formulae remain undetermined.
\end{abstract}

\section{Introduction}

In the last twenty years, there has been an increased interest in star polymers, a special class of branched polymers in which one end of each linear chain is tethered to a small central core to form a single molecule [1]. A particularly simple model for the static properties of an isolated star polymer ( $f$ arms, $N$ monomers per arm) in a solvent of low molecular weight was proposed by Daoud and Cotton 13 years ago [2]. This scaling picture was generalized by Raphaël et al. [3] to the case of a star polymer dissolved in a melt of linear chains of degree of polymerization $P<N$, which are chemically identical to the star arms. According to these authors, the $N$-monomer volume fraction, $\phi$, varies with the distance from the center of the star, $r$, as

$$
\begin{array}{rl}
\phi(r) \cong 1 & r<a f^{1 / 2} P^{1 / 4} \\
\phi(r) \cong P^{1 / 3} f^{2 / 3}(a / r)^{4 / 3} & a f^{1 / 2} P^{1 / 4}<r<R
\end{array}
$$

(*) Author for correspondence (e-mail: elie@ext.jussieu.fr)

$\left({ }^{* *}\right)$ U.R.A. 792 C.N.R.S.

(C) Les Éditions de Physique 1996 
where $a$ is the monomer size. The radius of the star, $R$, can be deduced from the condition $N f a^{3}=\int_{0}^{R} \mathrm{~d}^{3} r \phi(r)$. Using equations (1a) and (1b) one obtains $[3,4]$ :

$$
\begin{array}{rl}
R \cong a N^{3 / 5} f^{1 / 5} P^{-1 / 5} & N>f^{1 / 2} P^{3 / 4} \\
R \cong a N^{1 / 3} f^{1 / 3} & N<f^{1 / 2} P^{3 / 4}
\end{array}
$$

One should realize, however, that the results (1) and (2) cannot be valid for too small values of $f$. Indeed, in the limit of a linear molecule (i.e., for $f=1$ ), these results do not extrapolate to the well known results [5]: $R \cong a N^{3 / 5} P^{-1 / 5}$ for $P<N^{1 / 2}$, and $R \cong a N^{1 / 2}$ for $P>N^{1 / 2}$. It will be shown below that the results (1) and (2) are valid only for stars with a high enough number of arms $f>P^{1 / 2}$ In the present paper, we will therefore focus our attention on the reverse case $\left(f<P^{1 / 2}\right)$.

\section{Scaling Analysis}

Consider a star polymer made of $f$ identical arms, each arm consisting of $N$ monomers of size a. The star polymer is immersed in a melt of linear chains of degree of polymerization $P$, chemically identical to the star arms. We assume that $f<P^{1 / 2}$ Let us first consider what happens far away from the center. Following the idea of Daoud and Cotton [2], we describe each arm as a succession of growing spherical blobs. At a given distance $r$ from the center, there are $f$ blobs, one for each polymer chain. Since $f$ blobs cover a sphere of radius $r$, the blob radius is given by

$$
\xi(r) \cong r f^{-1 / 2}
$$

Within one blob, the arm behaves as an isolated chain and the number of monomers per blob, $g(r)$, is given by $\xi(r) \cong a g(r)^{3 / 5} P^{-1 / 5}$ The $N$-monomer volume fraction is given by

$$
\phi(r) \cong a^{3} g(r) \xi(r)^{-3} \cong P^{1 / 3} f^{2 / 3}(r / a)^{-4 / 3}
$$

Equations (3) and (4) are valid as long as the blob size $\xi$ is larger than the size of a melt blob, $l_{c}$ [5]. Consequently, these equations are valid only in the outer part of the star. This region, called $\mathrm{S} 2$, is defined by the condition $r>a P f^{1 / 2}$

For $r<a P f^{1 / 2}$, blobs from different chains overlap laterally (see Fig. 2) and their size $\Lambda$ is intermediate between and $r f^{-1 / 2}$ and $l_{\mathrm{c}}$ [6]. At a scale smaller than $\Lambda$, the arm behaves like an ideal chain of $g_{\Lambda} \cong(\Lambda / a)^{2}$ monomers. In order to calculate the subunit size $\Lambda$, we have to consider the perturbation parameter $\zeta \cong \frac{g_{\Lambda}(r)}{P} \phi(r)$ where $\phi(r) \cong a^{3} \frac{g_{\Lambda}(r)}{\Lambda(r)\left(r f^{-1 / 2}\right)^{2}}$ is the $N$-monomer volume fraction [6]. At a scale larger than $\Lambda$, the arm ceases to be ideal: the subunit size $\Lambda$ is therefore determined by the condition $\zeta \cong 1$. This leads to $g_{\Lambda}(r)=$ $P^{2 / 3} f^{-2 / 3}(r / a)^{4 / 3}$ and

$$
\Lambda(r)=a P^{1 / 3} f^{-1 / 3}(r / a)^{2 / 3}
$$

One can easily show that in this region, called S1 (see Fig. 1), the $N$-monomer volume fraction is still given by equation (4), although the structure of the blobs has changed.

When $\Lambda(r)$ becomes comparable to $r$, the $f$ blobs (corresponding to the $f$ arms of the star) overlap completely. This occurs for $r \cong a P f^{-1}$ For $r \leq a P f^{-1}$, the $N$-monomer volume fraction is thus simply given by $f$ times the volume fraction of a Gaussian chain:

$$
\phi(r) \cong f \frac{a}{r}
$$




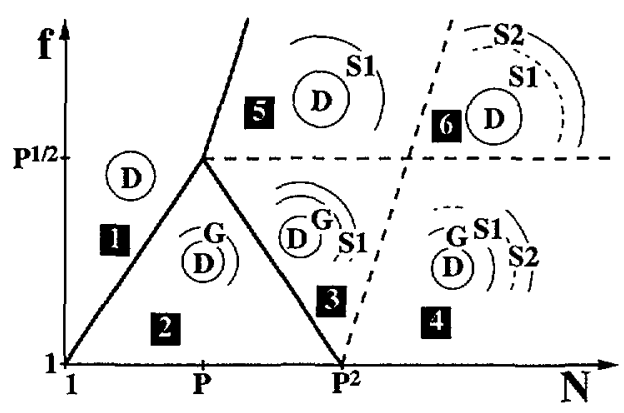

Fig. 1. - Various regimes for a star polymer ( $f$ arms, $N$ monomers per arm) dissolved in a melt of linear chains of degree of polymerization $P$, which are chemically identical to the star arms. The cross over between the different regions are given by: $1 / 2, N \cong f^{2} ; 2 / 3, N \cong P^{2} f^{-2} ; 3 / 4, N \cong P^{2} f^{1 / 2}$. The upper part of the figure corresponds to the case $f>P^{1 / 2}$ studied in reference [3]; the cross over between the different regions are given by: $1 / 5, N \cong P^{3 / 4} f^{1 / 2} ; 5 / 6, N \cong P^{2} f^{1 / 2}$

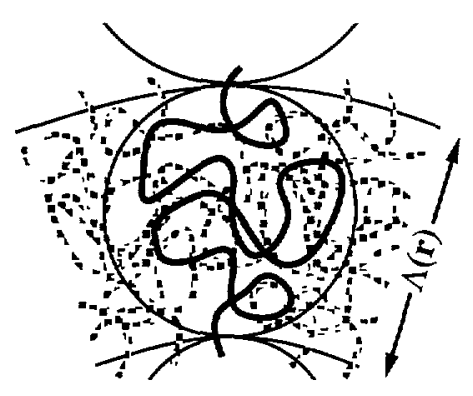

Fig. 2. - Schematic representation of the local structure of the star polymer in the region S1 $\left(a P f^{-1}<r<a P f^{1 / 2}\right)$. Each arm can be pictured as a succession of growing spherical blobs of size $\Lambda$ (intermediate between $r f^{-1 / 2}$ and $l_{c}$ ). Note that blobs from different chains overlap laterally.

For even smaller values of $r$, we reach a distance below which the $N$-monomer volume fraction is unity $(\phi \cong 1$ ). Using equation (6), we find that the radius of this dense region (called region D) is given by af. To summarize, a star with long arms $\left(N>P^{2} f^{1 / 2}\right)$ consists of four regions delimited by the radii $a f, a P f^{-1}$ and $a P f^{1 / 2}$

The radius of the star, $R$, may be obtained from the integral condition [7]

$$
N f a^{3}=\int_{0}^{R} \mathrm{~d}^{3} r \phi(r)
$$

Using equations (4) and (6) one obtains (Fig. 3)

$$
\begin{array}{rl}
R \cong a N^{3 / 5} f^{1 / 5} P^{-1 / 5} & N>P^{2} f^{-2} \\
R \cong a N^{1 / 2} & f^{2}<N<P^{2} f^{-2} \\
R \cong a N^{1 / 3} f^{1 / 3} & N<f^{2}
\end{array}
$$

The free energy of a star with $N>f^{2}$ can be written as the sum of two contributions due to the inner, dense region (the "core", $r<a f$ ) and the remaining part of the star (the "corona", af $<r<R): F_{\text {star }}=F_{\text {core }}+F_{\text {corona. }}$. The free energy of the corona is given by the $k T$ per 


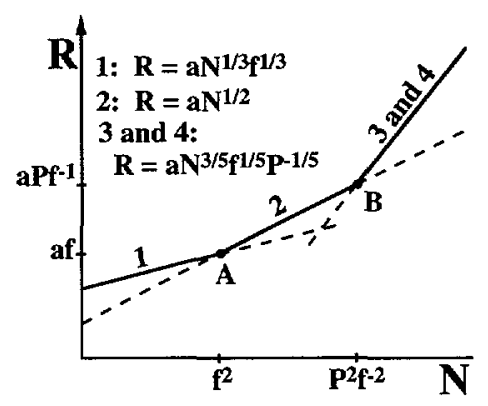

Fig. 3. - Variations of the star radius, $R$, as a function of the arm length, $N$. Note that for $N<f^{2}$, the star radius $a N^{1 / 3} f^{1 / 3}$ is greater than $a N^{1 / 2}$ (the arms are stretched with regard to their natural size). Numbers 1-4 refer to the corresponding regimes of Figure 1.

blob Ansatz

$$
\frac{F_{\text {corona }}}{k T} \cong f+\int_{a P f^{-1}}^{a P f^{1 / 2}} \frac{4 \pi r^{2} \mathrm{~d} r}{\Lambda(r)\left(r f^{-1 / 2}\right)^{2}}+\int_{a P f^{1 / 2}}^{R} \frac{4 \pi r^{2} \mathrm{~d} r}{\left(r f^{-1 / 2}\right)^{3}}
$$

where in the second term of the r.h.s the quantity $\Lambda(r)$ is given by equation (5). The first term corresponds to the free energy of the ideal parts of the arms (spatial region af $<r<a P f^{-1}$ ) Hence

$$
\begin{aligned}
F_{\text {corona }} / k T=f & f^{2}<N<P^{2} f^{-2} \\
F_{\text {corona }} / k T=N^{1 / 5} f^{7 / 5} P^{-2 / 5} & P^{2} f^{-2}<N<P^{2} f^{1 / 2} \\
F_{\text {corona }} / k T=f^{3 / 2}\left\{1+\ln \left(R /\left(a P f^{1 / 2}\right)\right)\right\} & N>P^{2} f^{1 / 2}
\end{aligned}
$$

The contribution $F_{\text {core }}$ accounts for the stretching penalty in the inner, dense region. A crude evaluation of $F_{\text {core }}$ may be obtained by assuming that for each arm the part of the chain inside the core $\left(f^{2}\right.$ monomers) is stretched to an end-to-end distance of the order of the core radius

$$
\frac{F_{\text {core }}}{k T} \cong f \frac{\left(a\left(f^{2}\right)^{1 / 3} f^{1 / 3}\right)^{2}}{a^{2}\left(f^{2}\right)} \cong f \quad N>f^{2}
$$

Note that for a star with $N<f^{2}$, the corona is absent and the core free energy is given by

$$
\frac{F_{\text {core }}}{k T} \cong f \frac{\left(a N^{1 / 3} f^{1 / 3}\right)^{2}}{a^{2} N} \cong f^{5 / 3} N^{-1 / 3} \quad N<f^{2}
$$

\section{Concluding Remarks}

(i) In this article, we have considered the static behavior of a star polymer ( $f$ arms, $N$ monomers per arm) immersed in a melt of linear chains ( $P$ monomers per chain) chemically identical to the star arms, assuming $f<P^{1 / 2}$ Our main result is the appearance of a Gaussian region within the star structure [8]. In particular, for $P^{2} f^{-2}<N<P^{2} f^{1 / 2}$, the star radius is given by $R \cong a N^{1 / 2}$ The origin of this result can be understood rather simply: since the star arms are immersed in a melt of chemically identical chains, they cannot be confined to a size smaller than their natural size $a N^{1 / 2}$ Since both the "dense star radius" $\left(a N^{1 / 3} f^{1 / 3}\right)$ and the "swollen 
star radius" $\left(a N^{3 / 5} f^{1 / 5} P^{-1 / 5}\right.$ ) would be smaller than $a N^{1 / 2}$ for $f^{2}<N<P^{2} f^{-2}$ (see Fig. 3), the star radius is given by $a N^{1 / 2}$ in this regime.

(ii) For $N<f^{2}$, the $N$-monomer volume fraction inside the star is of order unity: the star is dense. It is important to realize, however, that the star radius $a N^{1 / 3} f^{1 / 3}$ is greater than $a N^{1 / 2}$ : Due to steric hindrance (all the arms originate from the same point), the arms are stretched with respect to their natural size.

(iii) In the limit of a star with very few arms $(f \rightarrow 1)$, the results (8)-(10) extrapolate to the usual linear chain regimes: the chain is Gaussian $\left(R=a N^{1 / 2}\right)$ if $P>N^{1 / 2}$ and it is swollen $\left(R=a N^{3 / 5} P^{-1 / 5}\right)$ if $P<N^{1 / 2}$ (for $f=1$, the dense region is reduced to the size of a monomer)

(iv) For $\left(f=P^{1 / 2}\right)$, points $\mathrm{A}$ and $\mathrm{B}$ of Figure 3 coincide and the Gaussian region disappears. For $f>P^{1 / 2}$, one recovers all the results of reference [3].

(v) In this article we have considered the static properties of a single star polymer. Concentration effects will be published separately.

\section{Acknowledgments}

We thank M. Aubouy, G.H. Fredrickson and P. Pincus for useful discussions. We thank G. Grest for sending us manuscript [1] prior to publication.

\section{References}

[1] Grest G.S., Fetters L.J., Huang J.S. and Richter D., Adv. Chem. Phys., Vol. 94, E. Prigogine and S. A. Rice Eds. (New York, 1996).

[2] Daoud M., Cotton J.-P., J. Phys. France 43 (1982) 531.

[3] Raphaël E., Pincus P. and Fredrickson G.H., Macromolecules 26 (1993) 1996-2006.

[4] The results (1) and (2) are easily generalized to the case $P>N$; see the upper part of Figure 1.

[5] Consider an isolated linear chain, with degree of polymerization $N$, dissolved in a melt of shorter, chemically identical chains with degree of polymerization $P$. The chain behaves like a string of subunits, usually called melt blobs, each containing $g_{c}=P^{2}$ monomers. Within one melt blob the behavior is ideal, leading to a blob size $l_{c}=a P$. Different melt blobs repel each other, and the resulting self-avoiding chain (of unit step $l_{\mathrm{c}}$ ) has a size $R \cong\left(N / g_{c}\right)^{\nu} l_{c}$ where $\nu=3 / 5$ is the Flory 3 -dimensional exponent. See, e.g., de Gennes P.-G., "Scaling Concepts in Polymer Physics" (Cornell University Press, Ithaca, Fourth Printing, 1985).

[6] Aubouy M., Fredrickson G.H., Pincus P., Raphaël E., Macromolecules 28 (1995) 29792981.

[7] Note that the results concerning the star radius $R$ may be recovered by minimizing the Flory free energy per $\operatorname{arm} \frac{F_{\text {arm }}}{k T} \cong \frac{R^{2}}{N a^{2}}+N \frac{a^{3}}{P} c+N \frac{a^{6}}{P} c^{2}$ (where $c \cong N f / R^{3}$ is the average monomer concentration of the star), keeping in mind the conditions $a^{3} c \leq 1$ (i.e. $N f a^{3} \leq R^{3}$ ) and $R \geq a N^{1 / 2}$

[8] A somewhat similar Gaussian behavior was described by Birshtein and Zhulina for stars with a small number of semiflexible branches in low molecular weight solvents. See Birshtein T.M., Zhulina E.B., Polymer 25 (1984) 1453-1461. 